\title{
Qualidade da dieta de escolares de 7 a 10 anos do município de São Paulo: associação com o número e os locais de refeições
}

\author{
Diet quality in students aged 7 to 10 \\ years in São Paulo: Association \\ with the number and the \\ locations of the meals
}

Bárbara Grassi PRADO ${ }^{1}$

Patrícia de Fragas HINNIG $^{1}$

Luana Fiengo TANAKA ${ }^{1}$

Maria do Rosário Dias de Oliveira LATORRE ${ }^{1}$

\section{R E S U M O}

\section{Objetivo}

Analisar a relação entre a qualidade da dieta e o número e os locais de refeições de escolares de 7 a 10 anos do município de São Paulo.

\section{Métodos}

Trata-se de um estudo transversal, feito em 2013, com escolares de 7 a 10 anos de idade de duas escolas municipais de São Paulo. O consumo alimentar foi coletado a partir de três recordatórios de 24 horas, tendo sido calculado o Índice de Qualidade da Dieta Revisado. As médias de cada componente do índice foram analisadas segundo hábito de tomar café da manhã, número de refeições diárias, em casa, na escola e fora do lar.

\section{Resultados}

A maioria dos escolares ingeriu café da manhã, realizou menos de cinco refeições, nenhuma refeição na escola, três ou mais refeições em casa e nenhum consumo de refeições fora do lar. O hábito de tomar café da manhã

\footnotetext{
1 Universidade de São Paulo, Faculdade de Saúde Pública, Departamento de Epidemiologia. Av. Dr Arnaldo, 715, Cerqueira César, 01246-904, São Paulo, SP, Brasil. MRDO LATORRE. E-mail: <mdrddola@usp.br>.

Artigo elaborado a partir da tese de BG PRADO, intitulada "Qualidade da dieta de escolares do município de São Paulo". Universidade de São Paulo; 2014.
} 
apresentou associação com o maior consumo de vegetais e de leite e o maior número de refeições diárias com o menor consumo de sódio. O maior consumo de refeições em casa esteve associado ao menor consumo de gordura saturada, calorias de gordura sólida e açúcar e a melhor qualidade da dieta. As refeições fora do lar apresentaram associação com o maior consumo de cereais integrais e de calorias de gordura sólida e açúcar.

\section{Conclusão}

O maior consumo de alimentos saudáveis ocorre em casa, portanto ações de educação alimentar e nutricional devem incentivar o consumo de alimentos no lar e as escolhas alimentares saudáveis fora do lar.

Palavras-chave: Alimentação escolar. Crianças. Hábitos alimentares.

\section{A B S T R A C T}

\section{Objective}

To analyze the relationship between diet quality and meal number and location in school children aged 7-10 years in the city of São Paulo, Brazil.

\section{Methods}

This was a cross-sectional study of school children aged 7-10 years of two public schools in São Paulo, Brazil, in 2013. Food intake was investigated by three 24-hour recalls and diet quality was assessed by the Healthy Eating Index adapted for Brazil.

\section{Results}

Most schoolchildren had breakfast, fewer than five meals a day, no meal at school, three or more meals at home, and no meals away from home. Having breakfast was associated with higher intake of vegetables and milk, and higher number of daily meals was associated with lower sodium intake. The high prevalence of home meals was associated with lower intake of saturated fats, calories from fat, and sugar, and with higher diet quality. Meals away from home were associated with higher intake of whole grains, and calories from fat and sugar.

\section{Conclusion}

Higher intake of healthy foods takes place at home, so food and nutrition education activities should encourage food at home and healthy food choices away from home.

Keywords: Child nutrition. School feeding. Food habits.

\section{N T R O D U Ç Ã O}

A alimentação adequada é essencial para o desenvolvimento saudável da criança, e a disponibilidade de alimentos saudáveis, a companhia durante as refeições, o local e o número de refeições diárias apresentam associação com as escolhas alimentares da criança'-8.

A família tem papel importante na formação dos hábitos alimentares dos escolares e pode influenciar positiva ou negativamente no consumo alimentar da criança. A literatura mostra que quanto maior o número de refeições da criança com a família, principalmente em seu lar, maior o consumo de alimentos saudáveis, como frutas e verduras, em detrimento de lanches e refeições prontas para consumo, incluindo o refrigeran$\mathrm{te}^{2-4}$.

A prática dos pais e familiares de consumir o café da manhã pode aumentar significativamente o consumo dessa refeição pelas crianças ${ }^{5}$. Independentemente de ser realizada com os pais, a prática de realizar café da manhã está associada ao maior consumo de leite e derivados, frutas e cereais, e menor consumo de alimentos pouco saudáveis ${ }^{6}$. O maior número de refeições diárias encontra-se associado ao menor consumo de alimentos poucos saudáveis, e assim se apresenta como fator de proteção para obesidade ${ }^{7-8}$.

A escola também desempenha papel importante na formação dos hábitos alimentares da criança, que passa a realizar, pelo menos, uma 
refeição diária no ambiente escolar. No Brasil, as escolas públicas contam com o Programa Nacional de Alimentação Escolar (PNAE), que, implantado em 1955, é reconhecido pela United Nations Children's Found (Unicef) como o maior projeto de alimentação do mundo. É o mais antigo programa social do Governo Federal na área da Educação que, com caráter suplementar, visa contribuir para o crescimento, o desenvolvimento, a aprendizagem, o rendimento escolar dos estudantes e a formação de hábitos alimentares saudáveis, por meio da oferta da alimentação escolar e de ações de educação alimentar e nutriciona $^{9}$. A alimentação escolar representa um aspecto positivo na formação do hábito alimentar do escolar.

As refeições dos escolares, muitas vezes, ocorrem fora do lar, em restaurantes e lanchonetes. A alimentação fora do lar encontra-se associada ao maior consumo de refrigerantes, e menor consumo de frutas, hortaliças e leite. Estudos que analisaram os nutrientes da dieta de escolares observaram maior consumo de gordura total, gordura saturada e sódio, e menor consumo de fibras, ferro e cálcio entre os escolares que realizam maior quantidade de refeições fora do $\operatorname{lar}^{10-12}$.

Conhecer o consumo de alimentos pelo escolar em diferentes ambientes pode fornecer informações sobre a qualidade dos alimentos ofertados em cada local e o papel desses locais na promoção dos hábitos alimentares saudáveis dos escolares. O Índice de Qualidade da Dieta - Revisado (IQD-R) para a população brasileira é uma ferramenta importante que analisa a qualidade da dieta dos escolares, pois agrega a análise da quantidade e do tipo de alimentos e dos nutrientes consumidos, comparando-os com as recomendações nacionais ${ }^{13}$, e permite analisar a associação de diferentes variáveis relacionadas ao consumo alimentar.

Nesse contexto, o presente estudo teve como objetivo analisar a relação entre a qualidade da dieta e o número e os locais de refeição de escolares de 7 a 10 anos do município de São Paulo.

\section{M É T O D O S}

Trata-se de um estudo transversal, com escolares de 7 a 10 anos, em duas escolas municipais da região Oeste do município de São Paulo (SP). Em 2013, o projeto desta pesquisa foi apresentado a três escolas municipais da região Oeste do município de São Paulo. Somente duas escolas apresentaram interesse em participar da pesquisa, autorizando a realização do projeto. As escolas interessadas foram divididas em Escola 1 (período matutino) e Escola 2 (período vespertino). A seleção das escolas foi realizada por conveniência, uma vez que a pesquisa inicial objetivou analisar a qualidade da dieta de escolares expostos a fatores positivos para uma alimentação saudável, como a elevada escolaridade da pessoa de referência da família e a frequência à escola pública, que fornece alimentos com base em políticas públicas, como o Programa Nacional de Alimentação Escolar, que visa à promoção da alimentação saudável e à proibição, no ambiente escolar, do comércio de alimentos que geralmente oferece opções pouco saudáveis aos escolares.

No início do estudo, havia 207 crianças matriculadas na Escola 1 que estudavam do $2^{\circ}$ ao $4^{\circ}$ ano; 128 trouxeram assinado o Termo de Consentimento Livre e Esclarecido (TCLE), e, destas, uma criança foi excluída por ser especial, e 9 foram consideradas perdas, pois 8 não conseguiram responder ao Recordatório de 24 horas (R24h) e uma havia mudado de escola, restando 118 crianças com dados válidos.

Na Escola 2, 173 crianças estavam matriculadas do $2^{\circ}$ ao $4^{\circ}$ ano; 93 trouxeram assinado o TCLE; duas foram excluídas do estudo por serem especiais e 8 foram consideradas perdas, pois 7 não conseguiram responder ao R24h e uma mudou de cidade, restando 83 crianças com dados válidos. As duas escolas totalizaram 201 crianças, que constituíram a amostra final deste estudo. As informações sobre a caracterização da amostra do estudo estão na Figura 1. 


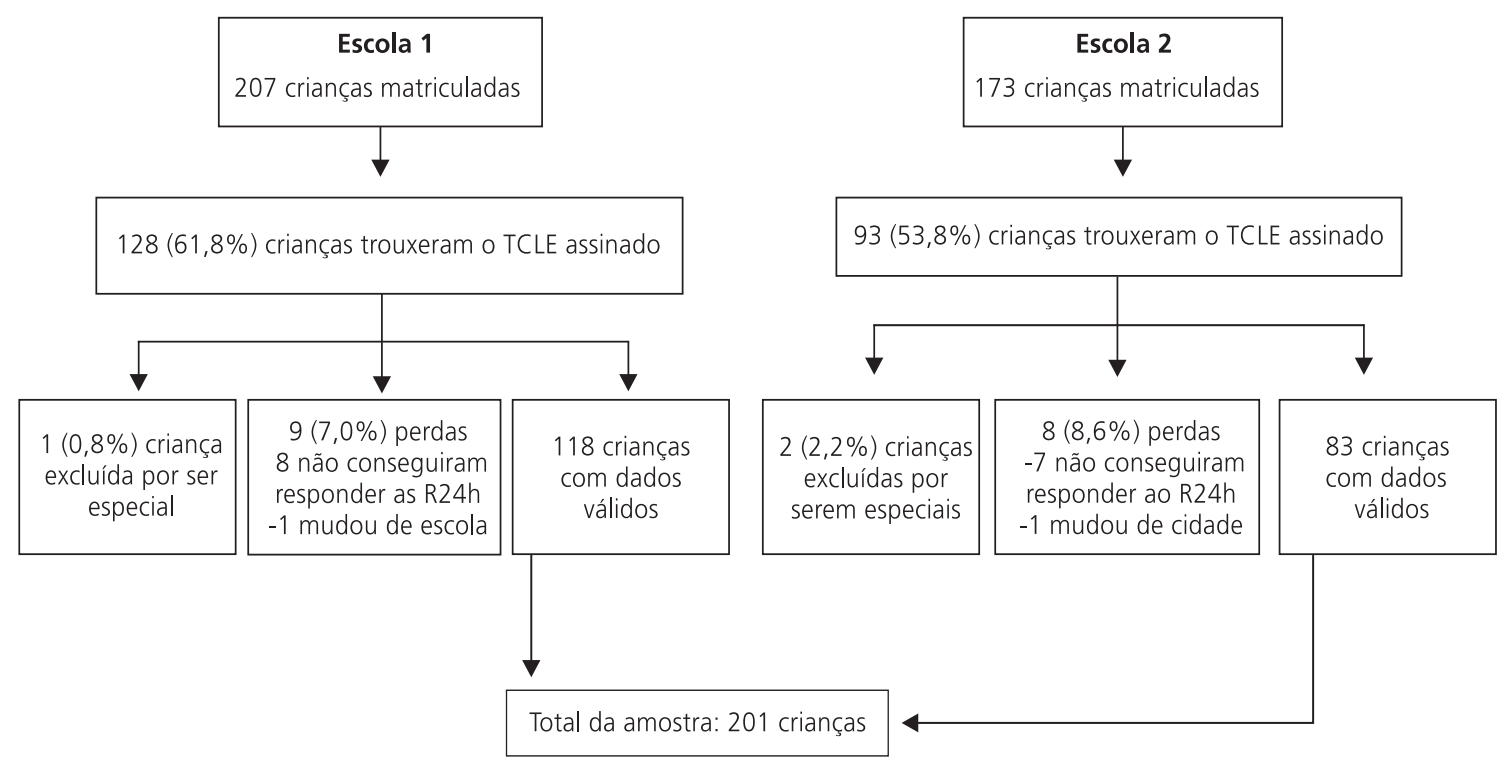

Figura 1. Caracterização da amostra do estudo.

Nota: TCLE: Termo de Consentimento Livre e Esclarecido; R24h: Recordatório de 24 horas.

As duas escolas não apresentaram diferença estatisticamente significativa em relação ao sexo, classificação socioeconômica e escolaridade da pessoa de referência da família, por isso foram analisadas em conjunto.

As coletas foram realizadas na própria escola, no período escolar. Inicialmente, foram realizadas as medidas antropométricas, seguidas pela aplicação do primeiro R24h. Com um intervalo de, aproximadamente, um mês, foi aplicado o segundo R24h, e, cerca de trinta dias depois, o último R24h, finalizando a coleta de dados. A aplicação dos R24h incluiu dois dias durante a semana ( $2^{\mathrm{a}}$ a $5^{\mathrm{a}}$ feira) e um dia do final de semana (domingo).

O recordatório de 24 horas foi aplicado por entrevistadores treinados com a finalidade de identificar e quantificar todos os alimentos e bebidas ingeridos pelos escolares no dia anterior à entrevista. Os alimentos do R24h foram transcritos e padronizados em um documento auxiliar; todos os alimentos foram convertidos em gramas $^{14}$, e as preparações culinárias foram desmembradas segundo seus ingredientes e quantidades. Para os cálculos relativos à quantidade de energia e macronutrientes dos alimentos, foi construído um banco de dados e elaboradas análises com adoção do programa Nutriquanti ${ }^{\circledR 15}$, e foram incluídos conteúdos de açúcar adicionado dos alimentos $^{16}$.

Para estimar a ingestão habitual dos nutrientes e grupos de alimentos, foi utilizada a plataforma online Multiple Source Method ${ }^{17}$. Na aplicação do método Multiple Source Method, os grupos de alimentos foram avaliados em quilocalorias (kcal). Para cada indivíduo, estimou-se a ingestão habitual de cada grupo de alimentos, que, posteriormente, foi utilizada para o cálculo do IQD-R e seus componentes. Considerou-se que os indivíduos apresentavam probabilidade de consumo habitual maior que zero para todos os grupos de alimentos e nutrientes.

A partir da estimativa de ingestão habitual dos nutrientes e grupos de alimentos, foi calculado o IQD-R ${ }^{13}$, método que analisa 12 componentes da dieta, atribuindo-Ihe uma pontuação que varia de zero a cem. Maiores pontuações do IQD-R representam dietas de melhor qualidade.

O Índice de Qualidade da Dieta - Revisado e seus componentes foram avaliados, separadamente, como variáveis quantitativas contínuas. 
As variáveis independentes analisadas foram a idade (anos), sexo (masculino; feminino), classificação socioeconômica (classe média a alta - classe A e B; classe baixa - $C$ e D) $)^{18}$, escolaridade da pessoa de referência da família (analfabeto ao ensino fundamental completo; ensino médio completo ao superior completo), total de dias que ingeriu café da manhã em 3 dias ( 0 a 1 dia; 2 e 3 dias), média de refeições por dia ( $<5 ; \geq 5$ refeições), média de refeições em casa por dia ( 0 a 2; 3 ou mais refeições), média de refeições na escola por dia (0; 1 ou mais refeições) e média de refeições fora do lar por dia (0; 1 ou mais refeições).

As variáveis foram descritas por meio de medidas de tendência central e de dispersão e frequências absoluta e relativa. A aderência à distribuição normal das variáveis quantitativas foi analisada por meio do teste Kolmogorov-Smirnov. Foi realizada a comparação de médias do IQD-R e seus componentes, segundo as variáveis independentes de consumo alimentar, utilizando o teste $t$ de Student ou Mann-Whitney. As análises foram realizadas no pacote estatístico Statistical Package for the Social Sciences (SPSS Inc., Chicago, Illinóis, Estados Unidos) versão 15.
Este projeto de pesquisa foi aprovado pelo Comitê de Ética em Pesquisa da Faculdade de Saúde Pública da Universidade de São Paulo sob o número de Protocolo n²337/2012.

\section{RES U LT A D O S}

A amostra total do estudo foi de 201 escolares: $54,7 \%$ eram do sexo feminino; $54,3 \%$ foram classificados na classe socioeconômica baixa; 55,4\% dos escolares apresentaram escolaridade da pessoa de referência da família com ensino médio completo ao ensino superior completo ou mais, e $77,8 \%$ das pessoas de referência da família eram as mães dos escolares.

A maioria dos escolares ingeriu o café da manhã em 2 a 3 dias do estudo (88,1\%). Mais de $60,0 \%$ dos escolares apresentaram consumo médio de refeições diárias abaixo de 5 refeições; $62,7 \%$ apresentaram consumo médio de nenhuma refeição na escola; 56,2\% apresentaram consumo médio diário de 3 ou mais refeições em casa, e 70,1\% nenhum consumo de refeições fora do lar.

Tabela 1. Estatística descritiva dos escores dos componentes do Índice de Qualidade da Dieta - Revisado (IQD-R) segundo o total de dias que ingeriu café da manhã em 3 dias e a média de refeições por dia. São Paulo (SP), 2013.

\begin{tabular}{|c|c|c|c|c|c|c|c|c|c|c|}
\hline \multirow{3}{*}{ Componentes } & \multicolumn{5}{|c|}{ Café da manhã } & \multicolumn{5}{|c|}{ Refeição por dia } \\
\hline & \multicolumn{2}{|c|}{ 0-1 dia } & \multicolumn{2}{|c|}{ 2-3 dias } & \multirow{2}{*}{$p^{*}$} & \multicolumn{2}{|c|}{$<5$} & \multicolumn{2}{|c|}{$\geq 5$} & \multirow{2}{*}{$p^{*}$} \\
\hline & $M$ & DP & M & DP & & M & DP & M & DP & \\
\hline Frutas totais & 3,56 & 1,99 & 3,49 & 1,53 & 0,292 & 3,37 & 1,63 & 3,69 & 1,49 & 0,227 \\
\hline Frutas integrais & 3,43 & 2,28 & 3,51 & 2,12 & 0,763 & 3,36 & 2,20 & 3,71 & 2,03 & 0,578 \\
\hline Vegetais totais & 4,29 & 1,40 & 4,67 & 1,09 & 0,051 & 4,66 & 1,11 & 4,57 & 1,19 & 0,615 \\
\hline Vegetais $^{* *}$ & 4,38 & 1,69 & 4,69 & 1,21 & 0,258 & 4,67 & 1,24 & 4,62 & 1,33 & 0,778 \\
\hline Cereais totais & 4,95 & 0,15 & 4,90 & 0,26 & 0,413 & 4,89 & 0,28 & 4,94 & 0,18 & 0,698 \\
\hline Cereais integrais & 0,34 & 1,04 & 0,53 & 1,16 & 0,324 & 0,45 & 1,15 & 0,59 & 1,14 & 0,118 \\
\hline Leite e derivados & 3,67 & 2,42 & 4,58 & 1,91 & 0,036 & 4,29 & 2,09 & 4,73 & 1,81 & 0,126 \\
\hline Carnes, ovos e leguminosas & 9,79 & 0,55 & 9,84 & 0,61 & 0,413 & 9,85 & 0,60 & 9,81 & 0,61 & 0,634 \\
\hline Óleos & 10,00 & 0,00 & 10,00 & 0,00 & 1,000 & 10,00 & 0,00 & 10,0 & 0,00 & 1,000 \\
\hline Gordura saturada & 6,99 & 1,73 & 7,43 & 1,43 & 0,208 & 7,25 & 1,54 & 7,57 & 1,34 & 0,134 \\
\hline Sódio & 1,46 & 1,40 & 1,69 & 1,42 & 0,443 & 1,46 & 1,41 & 1,99 & 1,38 & 0,003 \\
\hline Gord_AA $A^{* * *}$ & 9,54 & 2,31 & 9,43 & 2,71 & 0,902 & 9,65 & 2,68 & 9,14 & 2,61 & 0,257 \\
\hline IQD-R & 62,40 & 8,68 & 64,8 & 5,75 & 0,080 & 63,9 & 6,44 & 65,37 & 5,72 & 0,101 \\
\hline
\end{tabular}

Nota: *O teste Mann-Whitney foi utilizado para todos os componentes, com exceção do escore do componente leite e derivados e do IQD-R que foi utilizado o teste $t$ de Student; ${ }^{* *}$ Vegetais verdes-escuros, alaranjados e leguminosas; ${ }^{* * *}$ Calorias provenientes de Gordura Sólida e Açúcar de Adição.

M: Média; DP: Desvio-Padrão. 
A Tabela 1 apesenta a análise dos escores dos componentes do IQD-R segundo o consumo de café da manhã e número de refeições diárias. Os resultados apontam que os escolares que ingeriram café da manhã em 2 e 3 dias apresentaram média (4,67 pontos) estatisticamente maior $(p=0,051)$ na pontuação do componente vegetais totais quando comparados à pontuação média desse componente dos escolares que ingeriram café da manhã em 0 e 1 dia (4,29 pontos), mostrando que os escolares que apresentaram maior frequência de ingestão de café da manhã consomem mais vegetais.

Os escolares que ingeriram café da manhã em 2 e 3 dias apresentaram média (4,58 pontos) estatisticamente maior $(p=0,036)$ na pontuação do componente leite e derivados quando comparados à pontuação média deste componente dos escolares que ingeriram café da manhã em 0 e 1 dia (3,67 pontos), indicando maior consumo do componente leite e derivados entre os escolares com maior frequência de ingestão de café da manhã.

Quanto ao número de refeições diárias, os escolares que realizaram cinco ou mais re- feições por dia apresentaram média (1,99 pontos) estatisticamente maior $(p=0,003)$ na pontuação do componente sódio em relação à média deste componente (1,46 pontos) entre os escolares que realizaram menos de cinco refeições por dia, o que indica menor consumo de sódio entre os escolares com maior número de refeições diárias.

A Tabela 2 apresenta a análise dos escores dos componentes do IQD-R segundo o número de refeições realizadas em diferentes locais, incluindo a casa, a escola e fora do lar. Os escolares que consumiram três ou mais refeições em casa apresentaram média (7,57 pontos) estatisticamente maior $(p=0,004)$ na pontuação do componente gordura saturada quando comparados à pontuação média desse componente dos escolares que consumiram de 0 a 2 refeições em casa $(7,13$ pontos), o que indica menor consumo de gordura saturada pelos escolares que consumiram maior número de refeições em casa (Tabela 2).

Os escolares que consumiram três ou mais refeições em casa apresentaram média $(9,77$ pontos) estatisticamente maior, no limite da

Tabela 2. Estatística descritiva dos escores dos componentes do Índice de Qualidade da Dieta - Revisado (IQD-R) segundo refeições em casa, na escola e fora do lar. São Paulo (SP), 2013.

\begin{tabular}{|c|c|c|c|c|c|c|c|c|c|c|c|c|c|c|c|}
\hline \multirow{3}{*}{ Componentes } & \multicolumn{5}{|c|}{ Casa } & \multicolumn{5}{|c|}{ Escola } & \multicolumn{5}{|c|}{ Fora do lar } \\
\hline & \multicolumn{2}{|c|}{0 a 2} & \multicolumn{2}{|c|}{3 ou mais } & \multirow{2}{*}{$p^{*}$} & \multicolumn{2}{|c|}{0} & \multicolumn{2}{|c|}{1 ou mais } & \multirow{2}{*}{$p^{*}$} & \multicolumn{2}{|c|}{0} & \multicolumn{2}{|c|}{1 ou mais } & \multirow{2}{*}{$p^{*}$} \\
\hline & M & DP & M & DP & & M & $\mathrm{DP}$ & $\mathrm{M}$ & DP & & M & DP & M & DP & \\
\hline Frutas totais & 3,43 & 1,56 & 3,55 & 1,60 & 0,377 & 3,42 & 1,59 & 3,64 & 1,57 & 0,381 & 3,50 & 1,64 & 3,49 & 1,44 & 0,590 \\
\hline Frutas integrais & 3,39 & 2,20 & 3,58 & 2,09 & 0,566 & 3,36 & 2,20 & 3,72 & 2,01 & 0,627 & 3,60 & 2,10 & 3,26 & 2,22 & 0,260 \\
\hline Vegetais totais & 4,52 & 1,30 & 4,71 & 0,99 & 0,336 & 4,69 & 1,04 & 4,51 & 1,29 & 0,418 & 4,62 & 1,17 & 4,64 & 1,06 & 0,549 \\
\hline Vegetais $^{* *}$ & 4,54 & 1,44 & 4,73 & 1,13 & 0,297 & 4,72 & 1,15 & 4,53 & 1,46 & 0,310 & 4,61 & 1,34 & 4,75 & 1,10 & 0,476 \\
\hline Cereais totais & 4,89 & 0,26 & 4,92 & 0,24 & 0,193 & 4,93 & 0,21 & 4,87 & 0,31 & 0,113 & 4,91 & 0,26 & 4,90 & 0,23 & 0,271 \\
\hline Cereais integrais & 0,46 & 1,07 & 0,54 & 1,21 & 0,725 & 0,60 & 1,27 & 0,35 & 0,88 & 0,303 & 0,42 & 1,12 & 0,69 & 1,19 & 0,032 \\
\hline Leite e derivados & 4,42 & 1,79 & 4,50 & 2,14 & 0,781 & 4,42 & 2,11 & 4,55 & 1,78 & 0,661 & 4,42 & 2,09 & 4,58 & 1,75 & 0,591 \\
\hline Carnes ${ }^{* * *}$ & 9,74 & 0,78 & 9,91 & 0,41 & 0,072 & 9,85 & 0,63 & 9,81 & 0,56 & 0,505 & 9,87 & 0,45 & 9,75 & 0,86 & 0,543 \\
\hline Óleos & 10,00 & 0,00 & 10,00 & 0,00 & 1,000 & 10,00 & 0,00 & 10,00 & 0,00 & 1,000 & 10,00 & 0,00 & 10,00 & 0,00 & 1,000 \\
\hline Gordura saturada & 7,13 & 1,38 & 7,57 & 1,51 & 0,004 & 1,68 & 1,45 & 7,27 & 1,35 & 0,133 & 7,40 & 1,50 & 7,31 & 1,39 & 0,437 \\
\hline Sódio & 1,64 & 1,40 & 1,68 & 1,44 & 0,904 & 7,44 & 1,54 & 1,64 & 1,37 & 0,995 & 1,56 & 1,35 & 1,91 & 1,55 & 0,181 \\
\hline Gord_AA*** & 9,03 & 2,43 & 9,77 & 2,79 & 0,052 & 9,54 & 2,72 & 9,30 & 2,56 & 0,562 & 9,80 & 2,56 & 8,63 & 2,73 & 0,014 \\
\hline IQD-R & 63,22 & 6,54 & 65,46 & 5,75 & 0,011 & 64,65 & 6,06 & 64,20 & 6,45 & 0,623 & 64,72 & 6,36 & 63,91 & 5,79 & 0,399 \\
\hline
\end{tabular}

Nota: *O teste Mann-Whitney foi utilizado para todos os componentes, com exceção do escore do componente leite e derivados e do IQD-R que foi utilizado o teste $t$ de Student; ${ }^{* *}$ Vegetais verdes-escuros, alaranjados e leguminosas; ${ }^{* * *}$ Carnes, ovos e leguminosas; ${ }^{* * *}$ Calorias provenientes de gordura sólida e açúcar de adição.

M: Média; DP: Desvio-Padrão. 
significância ( $p=0,052)$, na pontuação do componente calorias provenientes de gordura sólida e açúcar de adição quando comparados à pontuação média desse componente dos escolares que consumiram de 0 a 2 refeições em casa $(9,03$ pontos), o que indica menor consumo de calorias provenientes de gordura sólida e açúcar de adição pelos escolares que consumiram maior número de refeições em casa.

A média da pontuação do IQD-R foi estatisticamente maior $(p=0,011)$ entre os escolares que consumiram três ou mais refeições em casa $(65,46$ pontos) quando comparada à média dos escolares que consumiram 0 a 2 refeições em casa $(63,22$ pontos), indicando que a qualidade da dieta é melhor entre aqueles que consumiram maior número de refeições em casa. Realizar refeições em casa pode ser considerado um fator de proteção para a melhor qualidade da dieta do escolar.

As análises das refeições realizadas na escola não apresentaram diferenças estatisticamente significativas nas médias dos componentes do IQD-R. A análise das médias dos componentes segundo a prática de consumo alimentar fora do lar mostrou que a média de pontos do componente cereais integrais foi estatisticamente maior $(p=0,032)$ entre os escolares que realizaram um ou mais refeições fora do lar ( 0,69 pontos) quando comparada à média dos escolares que não realizaram refeição fora do lar (0,42 pontos), mostrando que os escolares que realizaram refeições fora do lar apresentam maior consumo de cereais integrais.

Resultado inverso ao observado nas refeições realizadas em casa foi encontrado na análise do componente calorias provenientes de gordura sólida e açúcar de adição segundo a média de refeições fora do lar, em que a média de pontos deste componente foi estatisticamente maior $(p=0,014)$ entre os escolares que não realizaram refeições fora do lar (9,80 pontos) em relação aos escolares que realizaram um ou mais refeições fora do lar (8,63 pontos). Isso indica que os escolares que não realizaram refeições fora do lar tiveram menor consumo de calorias provenientes de gordura sólida e açúcar de adição. Portanto, realizar refeições fora do lar foi fator de risco para o maior consumo de gordura e açúcares.

\section{I SCUSS Ã O}

Este estudo - realizado com o objetivo de comparar as médias dos escores do IQD-R e de seus componentes com variáveis de consumo alimentar, que incluem a prática do café da manhã, número de refeições diárias e o local de refeição (casa, escola e fora do lar) -, foi conduzido em uma região do município considerada com bom poder aquisitivo. Os escolares estavam expostos a fatores positivos para uma alimentação saudável, como a elevada escolaridade da pessoa de referência da família, frequentar escola pública, que fornece alimentos com base no Programa Nacional de Alimentação Escolar ${ }^{9}$ e nas propostas de melhorias no cardápio do Departamento de Alimentação Escolar do município, que incluem a oferta de no mínimo duas refeições diárias e o aumento da oferta de alimentos saudáveis, como frutas e hortaliças ${ }^{19}$.

Assim, esperava-se observar bons resultados na análise da qualidade da dieta das crianças, principalmente no consumo de alimentos no ambiente escolar, entretanto as maiores pontuações de alguns componentes do IQD-R apresentaram relação com o hábito de tomar café da manhã, maior número de refeições diárias, maior consumo de alimentos em casa e nenhum consumo fora do lar.

Sobre a prática do café da manhã, neste estudo, a maioria dos escolares tinha o hábito de realizar o café da manhã $(88,1 \%)$, o que pode ser considerado elevado e positivo para os escolares, uma vez que essa prática está associada à melhoria na qualidade da alimentação das crianças $^{6}$. No Brasil, observou-se que cerca de 70,0 a 90,0\% dos escolares tinham o hábito de tomar café da manhã ${ }^{20}$, o que foi também observado entre escolares dos Estados Unidos e Europa ${ }^{21}$. 
O consumo do café da manhã pode auxiliar no controle do peso, maior ingestão de vitaminas e minerais e menor ingestão de gorduras e colesterol ${ }^{6}$. Também pode estar associado à melhoria do rendimento escolar, com efeito no desempenho cognitivo, memória, atenção e frequência escolar ${ }^{21-23}$.

O hábito de tomar café da manhã pode favorecer o consumo de alimentos que são fonte de cálcio, como os leites e derivados ${ }^{5,6}$. No presente estudo, observou-se que os escolares que consumiam café da manhã com maior frequência possuíam melhor qualidade da dieta e consumiam maior quantidade de leite e derivados. Esse fato reforça o papel das políticas públicas, que incentivam a prática cotidiana das crianças, em realizar essa refeição, e, assim, a formação de hábitos alimentares saudáveis para a vida adulta.

É importante destacar que os escolares do período matutino tinham a opção de realizar o café da manhã em casa ou na escola. O fato de a Prefeitura Municipal de São Paulo oferecer o café da manhã aos alunos é importante para a melhoria do desempenho escolar e para o aumento da oferta de leite e derivados, cereais e frutas: alimentos normalmente consumidos no café da manhã ${ }^{6,24}$.

Além da iniciativa da prefeitura de São Paulo, a literatura mostra que o hábito dos pais e familiares de tomar café da manhã também influencia esta prática entre os escolares ${ }^{5}$, por isso o hábito de tomar café da manhã em família deve ser incentivado.

No presente estudo, foi observada associação entre consumir café da manhã e apresentar maior consumo de vegetais totais. Sugere-se que o hábito de tomar café da manhã esteja associado a escolhas mais saudáveis ao longo do dia, pois gera sensação de saciedade no indivíduo, levando-o a reduzir o consumo de lanches energéticos, ricos em gordura, sódio e açúcares simples $^{6,24}$. Assim, acredita-se que a menor sensação de fome pode induzir o maior consumo de alimentos saudáveis, como os vegetais.
O maior número de refeições diárias também pode apresentar associação com hábitos alimentares saudáveis; isso foi observado no presente estudo: o maior número de refeições diárias esteve associado ao menor consumo de sódio. Como anteriormente mencionado, alguns estudos observaram que o maior número de refeições diárias gera menor sensação de fome ao longo do dia, tendo como consequência escolhas alimentares mais saudáveis, ou seja, menor consumo de alimentos ricos em gordura, sódio e açúcares, e maior consumo de frutas e hortaliças. Assim, realizar mais refeições por dia é fator de proteção para a obesidade ${ }^{6-8,24}$.

No presente estudo, a importância da família na alimentação do escolar foi analisada mediante o número de refeições em casa. Bem como na literatura, que aponta a família como promotora dos hábitos alimentares saudáveis ${ }^{2-4}$, foi observado que a maioria dos escolares realizava três ou mais refeições em casa, o que foi considerado como aspecto positivo.

O maior número de refeições em casa mostrou associação com o menor consumo de gordura saturada e de calorias provenientes de gordura sólida e açúcar de adição. Realizar mais refeições em casa apresentou-se como fator de proteção para uma melhor qualidade da dieta.

Portanto, nota-se que, em casa, as escolhas alimentares são mais saudáveis. Tanto a gordura saturada como as calorias provenientes de gordura sólida e açúcar de adição possuem como principais fontes alimentares os alimentos processados e os alimentos das redes de fast food. Em casa, esses alimentos, apesar de bastante utilizados, podem ser menos utilizados quando comparados aos outros ambientes, como as refeições realizadas em restaurantes e lanchonetes ${ }^{3-4}$.

Ao longo do dia do escolar, além das refeições realizadas em casa, as realizadas na escola ocorrem pelo menos duas vezes por dia. As escolas municipais de São Paulo oferecem, no mínimo, duas refeições diárias, podendo ser realizado um maior número de refeições quando em atividades extras. Entretanto, foi observado que a maioria 
dos escolares estudados apresentou consumo médio de nenhuma refeição na escola, o que foi considerado como baixo consumo de refeições na escola. Ainda, as análises das refeições realizadas na escola não apresentaram diferenças estatisticamente significativas nas médias dos componentes do IQD-R.

As propostas do PNAE como política pública ${ }^{9}$ e das melhorias de cardápio do Departamento de Alimentação Escolar ${ }^{19}$ do município de São Paulo são essenciais para a formação de hábitos alimentares saudáveis, mas o baixo consumo observado neste estudo aponta para a necessidade de explorar suas possíveis causas, que, de acordo com a literatura, podem estar associadas ao sabor das preparações e à inapetência do escolar 25,26 .

A alimentação fora do lar, que exclui as refeições realizadas em casa e na escola, é crescente entre os escolares ${ }^{10,27}$, entretanto a maioria dos estudados não consumiu alimentos fora do lar, o que foi considerado positivo, pois o consumo nessas condições encontra-se associado ao maior consumo de alimentos com alta densidade energética, riscos em gordura, açúcar e sódio, e menor consumo de alimentos saudáveis como frutas, hortaliças e leite ${ }^{10,12}$.

Entretanto, foi observado maior consumo de calorias provenientes de gordura sólida e açúcar de adição entre os escolares que realizaram refeições fora do lar, o que, possivelmente, se deva à elevada oferta de alimentos fontes desses nutrientes em restaurantes e lanchonetes, e baixa oferta de frutas e hortaliças ${ }^{10-12}$.

Foi observado maior consumo de cereais integrais entre os escolares que consumiram refeições fora do lar. Supõe-se que os restaurantes estejam ofertando preparações com grãos integrais e que essas preparações sejam bem aceitas pelos escolares. Assim, o baixo consumo de cereais integrais pode estar associado à indisponibilidade de acesso a esses alimentos pelos mesmos.

Além do incentivo à família, a escola também pode incentivar o consumo de cereais inte- grais por meio da oferta na merenda escolar. A prefeitura de São Paulo tem incluído em suas preparações alguns alimentos ou preparações a base de grãos integrais, sendo a aveia e o fubá (bolo ou polenta) exemplos de alimentos periodicamente ofertados $^{19}$. Pães e bolos preparados com farinhas integrais vêm sendo incorporados ao cardápio gradativamente, mas notou-se, no presente estudo, que esta oferta ainda não é suficiente. Se os escolares aceitam esses alimentos em refeições fora do lar, assim também pode ser nas escolas.

O papel de cada local de refeição no hábito alimentar da criança foi identificado. A preocupação central pauta-se no consumo de fast food e, portanto, de refeições fora do lar, pois possuem associação com o aumento da ingestão de gorduras, açúcares, sódio e redução do consumo de frutas, hortaliças, e leite e derivados ${ }^{10,11}$, apresentando relação direta com a qualidade da dieta dos escolares.

O consumo de maior número de refeições em casa foi considerado fator de proteção para o baixo consumo de gordura saturada, calorias provenientes de gordura sólida e açúcar de adição, e uma melhor qualidade da dieta. Portanto, as refeições realizadas em casa devem ser incentivadas, reduzindo-se o número de refeições fora do lar.

A literatura mostra que pesquisadores que realizaram atividades de educação alimentar e nutricional com escolares identificaram a importância de realizá-las com os escolares e sua família, pois, nessa faixa etária, os escolares estão expostos à alimentação oferecida pelos pais, que servem de exemplo para seus filhos ${ }^{28,29}$. Os resultados do presente estudo reafirmam essa importância por meio da observação de uma melhor qualidade da dieta em refeições realizadas em casa. A educação alimentar e nutricional para os escolares e familiares constitui estratégia importante na adoção permanente de hábitos alimentares saudáveis.

Segundo os resultados negativos em relação às refeições fora do lar observados no presente estudo, os restaurantes e lanchonetes também 
devem estar expostos às ações educativas que incluem capacitações sobre o aproveitamento integral dos alimentos, redução de gordura e sódio na alimentação, e a presença do nutricionista na elaboração de cardápios saudáveis. Para isso, políticas públicas deverão ser direcionadas a esses estabelecimentos com a finalidade de oferecer à população alimentos em condições higiênico-sanitárias satisfatórias e nutricionalmente adequadas. Vale ressaltar, que houve crescimento no mercado de alimentos prontos para consumo da linha saudável, mas estes alimentos ainda não são os mais consumidos fora do lar, muitas vezes por não constituir hábito da população ou serem inacessíveis financeiramente.

Considerando que tanto em casa como fora do lar os alimentos industrializados, principalmente os processados, são bastante utilizados, algumas ações políticas foram direcionadas para a indústria no intuito de reduzir a gordura, os açúcares e o sódio presentes nesses alimentos ${ }^{30-34}$.

A Organização Mundial de Saúde, baseada em diferentes experiências acerca da redução de sódio, açúcares e gorduras nos alimentos industrializados em países como Chile, Hungria, Tailândia, Reino Unido e Austrália, estabeleceu ações de incentivo à redução desses nutrientes nos alimentos produzidos pelo mundo. A meta é que, em 2020, tenha-se um teor mínimo desses nutrientes nos alimentos industrializados ${ }^{30}$.

É importante ressaltar que, dentre esses alimentos, estão alguns alimentos bastante consumidos pelo público infantil, como os salgadinhos de pacote, doces, bebidas lácteas, sucos prontos, bolos, biscoitos, macarrão instantâneo, embutidos e refeições prontas ${ }^{30-31}$.

Nas Américas, a Organização Pan-Americana de Saúde (OPAS) firmou um pacto de redução do percentual de gordura trans nos alimentos processados. As empresas devem respeitar o índice recomendado de, no máximo, 5\% de presença de gordura trans do total de gorduras em alimentos processados e $2 \%$ do total de gorduras em óleos e margarinas ${ }^{31}$.
No ano de 2014, a Organização Mundial da Saúde lançou a campanha "Action on Sugar", que possui como objetivo reduzir o consumo de açúcar pela população mundial e sugere a redução de até 30\% do açúcar em alimentos processados e redução do açúcar nas preparações doces. Ações educativas serão utilizadas como estratégia para educar a população sobre a importância dessa redução e sobre as formas de redução do consumo de açúcar na alimentação ${ }^{32}$.

No Brasil, a reformulação dos alimentos processados com a finalidade de reduzir o conteúdo de sódio, gordura saturada, gordura trans e açúcares, incentivando o consumo de alimentos in natura, constitui eixo do "Plano Nacional de Saúde 2012-2015" e do "Plano de Ações Estratégicas para o Enfrentamento das Doenças Crônicas Não-Transmissíveis no Brasil 2011-2022", do Ministério da Saúde ${ }^{33-35}$.

Todas essas estratégias tornam-se cruciais para que as refeições dentro e fora do lar e também nas escolas apresentem melhor qualidade nutricional.

\section{O N C L U S Ã O}

Ao analisar a relação entre a qualidade da dieta e o número e os locais de refeição de escolares de 7 a 10 anos do município de São Paulo, que possuem características essenciais para um hábito alimentar saudável, pode-se observar que essas variáveis influenciam a qualidade da dieta dos escolares. Assim, a prática de tomar o café da manhã, o maior número de refeições diárias, o maior número de refeições em casa e menor número de refeições fora do lar foram considerados como fator de proteção para uma melhor qualidade da dieta dos escolares.

Dessa forma, ações de educação alimentar e nutricional devem ser direcionadas aos escolares com intuito de incentivar o consumo de alimentos no lar e a redução do consumo de alimentos fora do lar. Além disso, políticas públicas devem ter como objetivo melhorar a qualidade nutricional dos alimentos industrializados e ofertados em restaurantes e lanchonetes. 


\section{COLABORADORES}

BG PRADO participou do desenho do estudo, coleta e análise dos dados e escrita do manuscrito. PF HINNIG participou do desenho do estudo, coleta e escrita do manuscrito. LF TANAKA participou da análise dos dados e escrita do manuscrito. MRDO LATORRE participou do desenho do estudo, análise e escrita do manuscrito.

\section{REFERÊ NCIAS}

1. Moreno LA, Rodríguez G. Dietary risk factors for development of childhood obesity. Curr Opin Clin Nutr Metab Care. 2007; 10(1):336-41. http://dx. doi.org/10.1097/MCO.0b013e3280a94f59

2. Haapalahti M, Mykkänen H, Tikkanen S, Kokkonen J. Meal patterns and food use in 10 to 11-year-old Finnish children. Public Health Nutr. 2003; 6(4):365-70. http://dx.doi.org/10.1079/phn2002433

3. Hammons AJ, Fiese BH. Is frequency of shared family meals related to the nutritional health of children and adolescents? Pedriatrics. 2011; 127(6):1565-74. http://dx.doi.org/10.1542/peds. 2010-1440

4. Mamum AA, Lawlor DA, O'Callagnan MJ, Williams GM, Najman JM. Positive maternal atitude to the family eating together decreases the risk of adolescente overweight. Obes Res. 2005; 13(8):1422-30. http://dx.doi.org/10.1038/oby. 2005

5. Pearson N, Biddle SJH, Gorely T. Family correlates of breakfast consumption among children and adolescents: A systematic review. Appetite. 2009; 52(1):1-7. http://dx.doi.org/10.1016/j.appet. 2008. 08.006

6. Utter J, Scragg R, Mhurchu C, Schaaf D. At-home breakfast consumption among New Zealand children: Associations with body mass index and related nutrition behaviors. J Am Diet Assoc. 2007; 107(4):570-6. http://dx.doi.org/10.1016/j.jada. 2007.01.010

7. Rech RR, Halpern R, Costanzi CB, Bergmann MLA, Alli LR, Mattos AP, et al. Prevalência de obesidade em escolares de 7 a 12 anos de uma cidade Serrana do RS, Brasil. Rev Bras Cineantropom Desempenho Hum. 2010; 12(2):90-7. http://dx.doi.org/10.5007/ 1980-0037.2010v12n2p90

8. Ortega RM, Redondo MR, Lopez-Sobaler AM, Quintas ME, Zamora MI, Andres $\mathrm{P}$, et al. Associations between obesity, breakfast-time food habits and intake of energy and nutrients in a group of elderly Madrid residents. J Am Coll Nutr. 1996;
15(1):65-72. http://dx.doi.org/10.1080/07315724. 1996.10718566

9. Brasil. Ministério da Saúde. Resolução/CD/FNDE $n^{\circ}$ 38 de 16 de julho de 2009. Dispõe sobre o atendimento da alimentação escolar aos alunos da educação básica no Programa Nacional de Alimentação Escolar (PNAE). Diário Oficial da União. 2009; 16 jun.

10. French SA, Story M, Neumark-Sztainer D, Fulkerson JA, Hannan P. Fast food restaurant use among adolescents: Associations with nutriente intake, food choices and behavioral and psychosocial variables. Int J Obes Relat Metab Disord. 2001; 25(12):1823-33. http://dx.doi.org/10.3402/fnr. v59.26488

11. Lin BH, Guthrie J, Frazao E. Quality of children's diets at and away from home: 1994-96. Food Rev. 1999; 22(1):2-10.

12. Taveras EM, Berkey CS, Rifas-Shiman SL, Ludwig DS, Rockett HRH, Field $A E$, et al. Association of consumption of fried food away from home with body mass index and diet quality in older children and adolescents. Pediatrics. 2005; 116(4):518-24. http://dx.doi.org/10.1542/peds.2004-2732

13. Previdelli AN, Andrade SC, Pires MM, Ferreira SRG, Fisberg RM, Marchioni DM. Índice de Qualidade da Dieta Revisado para população brasileira. Rev Saúde Pública. 2011; 45(4):794-8. http://dx.doi. org/10.1590/S0034-89102011005000035

14. Pinheiro ABV, Lacerda EMA, Benzecry EH, Gomes MCS, Costa VM. Tabela para avaliação de consumo alimentar em medidas caseiras. São Paulo: Atheneu; 2004.

15. Galante AP. Desenvolvimento e validação de um método computadorizado para avaliação do consumo alimentar, preenchido por indivíduos adultos utilizando a Web [doutorado]. São Paulo: Universidade de São Paulo; 2007.

16. Instituto Brasileiro de Geografia e Estatística. Pesquisa de orçamentos familiares 2008/2009: tabelas de composição nutricional dos alimentos consumidos no Brasil. Rio de Janeiro: IBGE; 2011.

17. German Institute of Human Nutrition PotsdamRehbrucke, Departament of Epidemiology. The multiple source method. Potsdam: German Institute of Human Nutrition Potsdam-Rehbrucke; 2007 [cited 2015 Nov 20]. Available from: https://nugo. dife.de/msm

18. Associação Brasileira de Empresas de Pesquisa. Critério de classificação econômica do Brasil. São Paulo: Abep; 2011.

19. São Paulo. Decreto $n^{\circ} 35.458$, de 31 de Agosto de 1995. Diário Oficial do Município de São Paulo. 1995; 1 set, p.1. 
20. Sturion GL, Silva MV, Ometto AMH, Furtuoso COM, Pipitone MAP. Fatores condicionantes da adesão dos alunos ao Programa de Alimentação Escolar no Brasil. Rev Nutr. 2005; 18(2):167-81. http://dx. doi.org/10.1590/S1415-52732005000200001

21. Rampersaud GC, Pereira MA, Girard BL, Adams J, Metzl J. Breakfast habits, nutritional status, body weight, and academic performance in children and adolescents. J Am Diet Assoc. 2005; 105(5):743-60. http://dx.doi.org/10.1016/j.jada.2005.02.007

22. Affenito SG. Breakfast: A missed opportunity. J Am Diet Assoc. 2007; 107(4):565-9. http://dx.doi.org/ 10.1016/j.jada.2007.01.011

23. Benton D, Jarvis $M$. The role of breakfast and a mid-morning snack on the ability of children to concentrate at school. Physiol Behav. 2007; 90(23):382-5. http://dx.doi.org/10.1016/j.physbeh. 2006.09.029

24. Nicklas TA, Baranowsky T, Cullen KW, Berenson G. Eating patterns, dietary quality and obesity. J Am Coll Nutr. 2001; 20(6):599-608. http://dx.doi.org/ 10.1080/07315724.2001.10719064

25. Teo CRPA, Corrêa EM, Gallina LS, Fransozi C. Programa nacional de alimentação escolar: adesão, aceitação e condições de distribuição de alimentação na escola. Nutrire. 2009; 34(3):165-85. http:// dx.doi.org/10.1590/S1415-52732007000300007

26. Danelon MAS, Danelon MS, Silva MV. Serviços de alimentação destinados ao público escolar: análise da convivência do Programa de Alimentação Escolar e cantinas. Segur Aliment Nutr. 2006; 13(1):85-94. http://dx.doi.org/10.5007/1980-0037.2010V12 N4P221

27. Nielson SJ, Siega-Riz AM, Popkin BM. Trends in food locations and sources among adolescents and young adults. Prev Med. 2002; 35(2):107-13. http:// dx.doi.org/10.1006/pmed.2002.1037
28. Philippi ST, Laterza AR, Cruz ATR, Ribeiro LC. Pirâmide alimentar adaptada: guia para escolha dos alimentos. Rev Nutr. 1999; 12(1):65-80. http://dx. doi.org/10.1590/S1415-52731999000100006

29. Prado BG, Guimarães LV, Lopes MAL, Bergamaschi DP. Efeito de ações educativas no consumo de alimentos no ambiente escolar. Nutrire. 2012; 37(3):281-92. http://dx.doi.org/10.4322/nutrire. 2012.022

30. Verstraeten $R$, Roberfroid D, Lachat C, Leroy JL, Holdsworth M, Maes L, et al. Effectiveness of preventive school-based obesity interventions in low- and middle-income countries: A systematic review. Am J Clin Nutr. 2012; 96(2):227-8. http:// dx.doi.org/10.3945/ajcn.112.035378

31. World Health Organization. Creating an enabling strategies: Report of Joint Technical Meeting held by WHO and FSA/UK. Genebra: WHO; 2010.

32. Organização Pan-Americana da Saúde. Américas livres de gorduras trans: conclusões e recomendações. Washington (DC): OPAS; 2007.

33. Consensus Action on Salt and Health. Action on sugar. London: University of London; 2014.

34. Brasil. Ministério da Saúde. Secretaria de Vigilância em Saúde. Plano de ações estratégicas para o enfrentamento das Doenças Crônicas Não Transmissíveis (DCNT) no Brasil 2011-2022. Brasília: Ministério da Saúde; 2011.

35. Brasil. Ministério da Saúde. Plano Nacional de Saúde - PNS: 2012-2015. Brasília: Ministério da Saúde; 2011.

Recebido: julho 14, 2014

Versão final: julho 16, 2015 Aprovado: agosto 3, 2015 